\title{
A Simulation of MR Imaging Sequences Using the Numerical Solutions of Bloch Equations
}

Pin-Pin Hsu, Jyh-Horng Chen

Department of Electrical Engineering, National Taiwan University, Taipei, Taiwan, R.O.C.

\begin{abstract}
We design a computer simulation program to solve the Bloch Equations. By applying different RF pulse sequences, we can simulate $M R$ images and their imaging contrasts for material with different $\mathrm{T} 1$ and $\mathrm{T} 2$. The imaging contrast fits well with the calculated formula value. For a RF pulse sequence does not have a signal close form intensity, we can use this program to simulate the images and their $\mathrm{T} 1, \mathrm{~T} 2$ contrasts.
\end{abstract}

\section{Introduction}

Many RF pulse sequences have their close form formulae of signal intensity. But not every RF pulse sequence has such a formula. Using this simulation program we can find the signal amplitude and imaging contrast properly. Here we apply it in typical RF pulse sequences : CPMG experiment, Stimulated Echo [1], Conventional Spin Echo Imaging, Conventional Gradient Echo Imaging, Gradient-Recalled Acquisition in the Steady State Imaging (GRASS) [2] and Stimulated Echo Acquisition Mode ( STEAM) Imaging [1].

\section{Method}

The Bloch Equations in the rotating frame are :

$\frac{d M_{x}}{d t}=\gamma\left(M_{y} \Delta B_{0}-M_{z} B_{1}\right)-\frac{M_{x}}{T_{2}}$

$\frac{d M_{y}}{d t}=\gamma\left(-M_{x} \Delta B_{0}+M_{z} B_{1 x}\right)-\frac{M_{y}}{T_{2}}$

$\frac{d M_{z}}{d t}=\gamma\left(M_{x} B_{1 y}-M_{y} B_{1 x}\right)-\frac{M_{z}-M_{0}}{T_{1}}$

where $\mathrm{Mx}, \mathrm{My}, \mathrm{Mz}$ is the magnetization of $\mathrm{x}, \mathrm{y}$, and $\mathrm{z}$ axes, respectively. $\gamma$ is $4257 \mathrm{~Hz}$ /gauss, $\Delta B_{10}$ is the field difference from the mainly magnetic field $B_{0}$, and $B_{1 x}, B_{1 v}$ is the RF pulse on $x$ and $y$ axis, respectively. Here we use RK 4th method to solve these differential equations.

In this simulation we assume that we are in the ideal situation, i.e. : rectangular gradient wave forms, no field inhomogeneity and perfect refocusing RF pulse.

The parameters of each RF pulse sequence are given in Table 1. and Table 2. The variable $h$ is the time resolution in the RK 4th method. $\alpha$ o RF amplitude in these pulse sequence can be calculated as $\alpha^{\circ}=0.0587^{*} 5^{*} 10^{-6 *} \alpha /\left(h^{*} 90\right)$ gauss.

In CPMG experiment, we use 19 spins in simulation. The four echo simulated result is given in Fig. 1.
In Stimulated Echo ( STE ), the simulated spin number is 299. The RF pulse sequence is $90^{\circ}{ }_{x}-5 m s-90^{\circ} x-15 m s-90^{\circ}$. Fig. 2. shows the simulation echo trains.

In imaging simulation, there are two phantoms with the same proton density for imaging, the left one, phantom $A$ and the right one, phantom $B$. The image sizes are all $64 \times 64$.

The simulated image for Conventional Spin Echo is given in Fig. 3. The signal intensity formula is given as,

$S \propto\left[1-2 \exp \left(-\frac{T R-\frac{T E}{2}}{T_{1}}\right)+\exp \left(-\frac{T R}{T_{1}}\right)\right] \exp \left(-\frac{T E}{T_{2}}\right)$

Figure 4. shows the simulated image of Conventional Gradient Echo. The signal intensity is,

$S \propto\left(1-\exp \left(-\frac{T R}{T_{1}}\right)\right)^{*} \exp \left(-\frac{T E}{T_{2}^{*}}\right)$

Here we set $\mathrm{T}^{*}{ }_{2}=\mathrm{T}_{2}$.

In the GRASS imaging, the flip angle $\alpha$ is $45^{\circ}$. After the read gradient, we place a rewinder gradient on the phaseencoding axis to undo the phase shift induced by the phaseencoding gradient. The result is in Fig. 5 . The signal intensity can be shown as,

$$
S \propto \frac{\sin (\alpha)}{1+\frac{T_{1}}{T_{2}}-\cos (\alpha) *\left(\frac{T_{1}}{T_{2}}-1\right)}
$$

In STEAM Imaging, the RF pulse sequence is $90^{\circ}{ }^{-5.5 m s-}$ $90^{\circ} x^{-46.5 m s-90^{\circ}} x^{-6.5 m s-E c h o . ~ T h e ~ p h a s e-e n c o d i n g ~ g r a d i e n t ~ i s ~}$ turned on after the third $90^{\circ}$ x pulse. The simulated image is shown in Fig. 6.

\begin{tabular}{|l|l|l|l|l|}
\hline & h (ms) & T1 (ms) & T2 (ms) & $\begin{array}{l}\text { Gx } \\
\text { (gauss } / \mathrm{cm})\end{array}$ \\
\hline CPMG & 0.005 & 200 & 80 & 0.02 \\
\hline STE & 0.0005 & 200 & 80 & 0.015 \\
\hline
\end{tabular}

Table 1.

\section{Results and Discussion}

Refocusing Time In complicated MR sequence, echo time is not trivial to predict. For burst imaging and stimulated-echo based sequences, we show that simulation results can give exact echo time as one expects ( Fig. 2).

Image Contrast The imaging contrast results are given in Table 3. These simulated contrasts fit well with the calculated values. STEAM imaging sequence, as one expects, shows 


\begin{tabular}{|l|l|l|l|l|l|l|l|l|l|}
\hline & h (ms) & TR (ms) & TE (ms) & Phantom A & Phantom B & $\begin{array}{l}\text { Gx } \\
\text { (gauss/cm) }\end{array}$ & $\begin{array}{l}\text { Gy } \\
(\mathrm{gaus} / \mathrm{cm})\end{array}$ & $\begin{array}{l}\text { resolution } \\
\text { of } x(\mathrm{~mm})\end{array}$ & $\begin{array}{l}\text { resolution } \\
\mathrm{of} y(\mathrm{~mm})\end{array}$ \\
\hline $\mathrm{SE}$ & 0.005 & 400 & 60.5 & $\begin{array}{l}\mathrm{T} 1=200 \mathrm{~ms} \\
\mathrm{~T}=100 \mathrm{~ms}\end{array}$ & $\begin{array}{l}\mathrm{T} 1=100 \mathrm{~ms} \\
\mathrm{~T} 2=50 \mathrm{~ms}\end{array}$ & 0.5 & 0.0015 & 0.91 & 0.96 \\
\hline GE & 0.005 & 400 & 53.7 & $\begin{array}{l}\mathrm{T} 1=200 \mathrm{~ms} \\
\mathrm{~T} 2=100 \mathrm{~ms}\end{array}$ & $\begin{array}{l}\mathrm{T} 1=100 \mathrm{~ms} \\
\mathrm{~T} 2=50 \mathrm{~ms}\end{array}$ & 0.5 & 0.0015 & 0.91 & 0.96 \\
\hline GRASS & 0.0005 & 21 & 10.49 & $\begin{array}{l}\mathrm{T} 1=100 \mathrm{~ms} \\
\mathrm{~T} 2=50 \mathrm{~ms}\end{array}$ & $\begin{array}{l}\mathrm{T} 1=150 \mathrm{~ms} \\
\mathrm{~T} 2=50 \mathrm{~ms}\end{array}$ & 0.1 & 0.00312 & 2.29 & 2.29 \\
\hline STEAM & 0.0005 & 1352 & 58.5 & $\begin{array}{l}\mathrm{T} 1=200 \mathrm{~ms} \\
\mathrm{~T} 2=50 \mathrm{~ms}\end{array}$ & $\begin{array}{l}\mathrm{T} 1=80 \mathrm{~ms} \\
\mathrm{~T} 2=50 \mathrm{~ms}\end{array}$ & 0.1 & 0.012 & 2.35 & 2.45 \\
\hline
\end{tabular}

T1-weighted contrast behavior.

Limitation The solutions will converge under certain imaging parameter ranges. These parameters affecting phase angle should be choose carefully. Dynamic RK method is developed to increase efficiency of simulation.

For further study, we are simulating more new RF pulse sequences such as Burst imaging. Also, trade-off between simulation spin number and computing time will be carefully optimized.

\begin{tabular}{|l|l|l|l|}
\hline $\mathrm{S}_{\mathrm{A}}: \mathrm{S}_{\mathrm{B}}$ & $\begin{array}{l}\text { Theoretical } \\
\text { Value }\end{array}$ & $\begin{array}{l}\text { Simulated } \\
\text { Value }\end{array}$ & Error Ratio \\
\hline Spin Echo & $1.55: 1$ & $1.53: 1$ & $-1.29 \%$ \\
\hline Gradient Echo & $1.51: 1$ & $1.50: 1$ & $-0.6 \%$ \\
\hline GRASS & $1.13: 1$ & $1.16: 1$ & $2.65 \%$ \\
\hline STEAM & & $1.25: 1$ & \\
\hline
\end{tabular}

Table 3.

\section{Reference}

[1] J. Frahm, et. al, "Stimulated Echo Imaging.", J. Magn. Reson. 64, 81-93 (1985).

[2] Felix W. Wehrli, "Fast-Scan Magnetic Resonance Principles and Applications", Raven Press, New York, 1991.

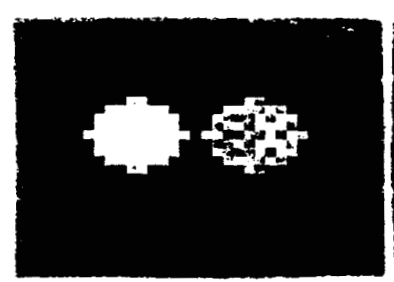

Fig. 3 Spin Echo Imaging

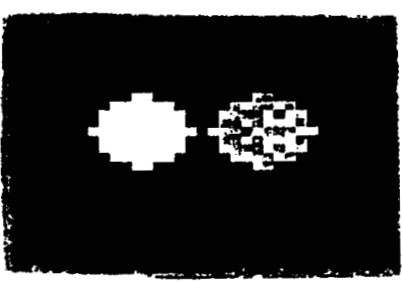

Fig. 4 Gradient Echo Imaging
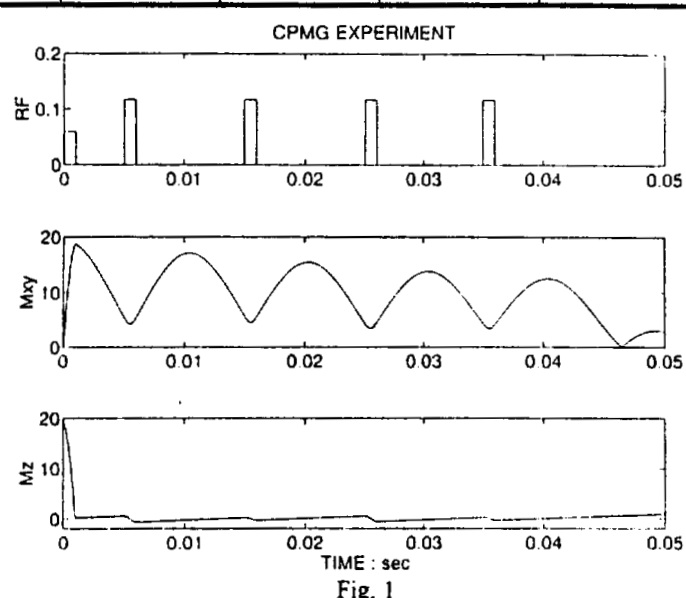

Fig. 1
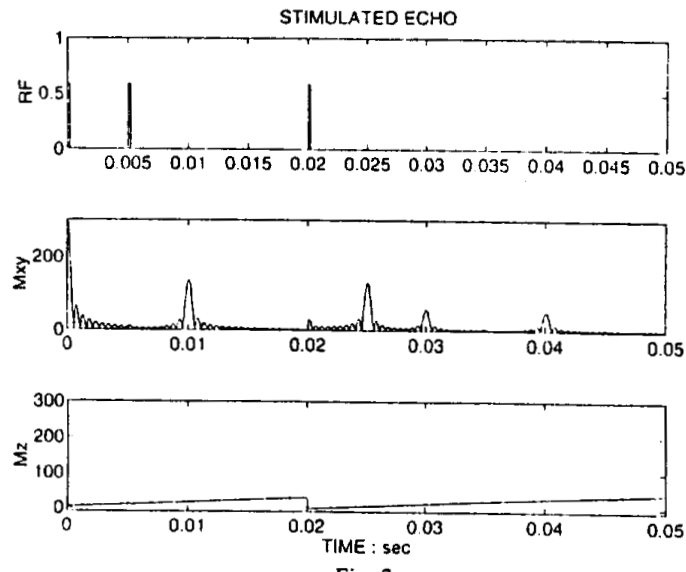

Fig. 2

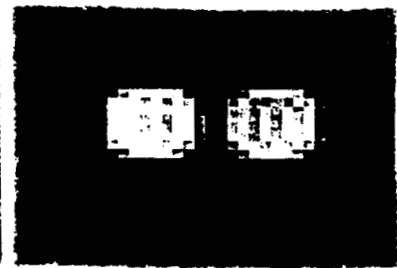

Fig. 5 GRASS Imaging

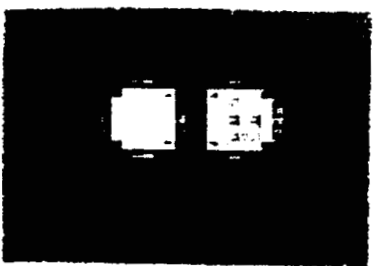

Fig. 6 STEAM Imaging 